\title{
Too Much of a Good Thing: Cancer Screening in the Old and Infirm
}

\author{
Douglas J. Robertson, MD, MPH ${ }^{1,2,3}$ \\ ${ }^{1}$ Section of Gastroenterology (111E), Department of Veterans Affairs Medical Center, White River Junction, VT, USA; ${ }^{2}$ Dartmouth Medical \\ School, Hanover, NH, USA; ${ }^{3}$ The Dartmouth Institute, Hanover, NH, USA.
}

$J$ Gen Intern Med 28(1):6-8

DOI: $10.1007 / \mathrm{s} 11606-012-2233-z$

(c) Society of General Internal Medicine 2012

I $\mathrm{t}$ is recruiting season for Gastroenterology Fellowship programs. In reading their personal statements, I have noted that prospective applicants often highlight one of the major draws of our subspecialty - treating life-threatening emergencies. Massive hematemesis and obstructing volvulus are high-stakes and high-reward opportunities that can raise the catecholamine level of even the most seasoned endoscopist. Success in such cases provides an extremely high level of satisfaction. While failure or even endoscopic complication in these cases is disappointing, the result is that the patient undergoes surgery that they likely would have needed if the endoscopy had not been attempted. That is why my heart rate and blood pressure are never higher than when a routine screening colonoscopy appears headed to complication. While thankfully rare, the thought of subjecting an individual without prior symptoms or problems to a hospitalization or surgery is extremely distressing. So, taken from that perspective, screening colonoscopy is the highest risk game in town and that is exactly why the decision to screen is such a critical one.

The paper by Haggstrom and colleagues in this issue of JGIM $^{1}$ suggests that there is room for improvement in decision making regarding colorectal cancer screening. They surveyed 1,975 physicians representing family medicine, internal medicine and obstetrics and gynecology with a high degree of success (nearly $70 \%$ compliance). Across a range of ages and comorbidities, they asked respondents the test or combination of tests most likely to be recommended for screening, including the option not to screen. Enthusiasm for screening was quite strong across the panel of vignettes, even in circumstances where screening likely offers little or no benefit. For example, $71 \%$ of those surveyed recommended screening an 80-yearold with significant symptomatic ischemic cardiomyopathy.

The contents of this work do not represent the views of the Department of Veterans Affairs or the United States Government

Published online September 28, 2012
Even more striking was the level of enthusiasm for screening those with unresectable non-small cell lung cancer. In fact, $25 \%$ of respondents recommended screening an 80-year-old in such a circumstance.

The high response rate of the survey is one of the notable strengths of the paper. This likely reflects the clean layout of the vignettes using three discrete age groups (50, 65 and 80) and three separate patient types (healthy, cardiomyopathy and lung cancer). Given the straightforward design, I suspect that respondents fully understood what they were being asked and that the results accurately reflect clinician's recommendations in such cases. Another important feature of the work is that they asked clinicians not only if they would screen, but what modality they would use. Current screening guidelines offer a panel of options including noninvasive tests (like fecal occult blood tests) and structural exams (like colonoscopy). ${ }^{2-4}$ Interestingly they found that clinicians tended to choose less invasive strategies for the older and sicker patients in the vignettes. For example, physicians recommended fecal occult blood test (FOBT) alone in only $5 \%$ of healthy 50 -year olds, but chose FOBT as the first choice in $20 \%$ of healthy 80 -year olds. By having the physicians commit to a specific screening strategy the survey gained further insights into behaviors that might offer opportunity for improvement.

While interesting and important, the paper does have some significant shortcomings. The most obvious is the use of survey methodology. Of course, some might argue that relying on vignettes does not reflect actual real world practice. In fact, I think that is unlikely to be the case. There is outstanding evidence from studies with other designs that there is a tendency in practice to be overzealous with screening. This was shown most dramatically in one study combining Behavioral Risk Factor Surveillance Systems (BRFSS) data regarding Pap smears in combination with the direct survey of patients who had undergone hysterectomy. It was estimated the nearly half the women with hysterectomy were still being screened with PAP smears unnecessarily. ${ }^{5}$ There is also evidence in the colorectal cancer screening literature that screening is likely overutilized in sick patients that are least likely to benefit. Using administrative data derived from the Veterans Administration and Medicare, Walter and colleagues showed that 
screening rates determined by procedural coding were above $40 \%$ for those with severe comorbidities and life expectancies of less than 5 years. ${ }^{6}$

Another mentionable limitation is the somewhat dated nature of the survey itself, it having been administered in late 2006 and early 2007. The survey significantly predated the updated 2008 guidelines from the United States Preventive Services Task Force. ${ }^{3}$ That release of the guidelines explicitly suggests that screening above age 75 "may not be recommended". Of course, it is possible that this guideline may now be tempering enthusiasm for screening in older age groups. However, my guess is that the rate would still be reasonably high, since in the current paper, screening of healthy octogenerians was recommended by $90 \%$ of clinicians. There have been other developments in the arena of colorectal cancer screening since the survey occurred. Most importantly, there have been a number of publications citing the effectiveness (in large randomized trials) of sigmoidoscopy ${ }^{7-9}$ and papers questioning the effectiveness of colonoscopy, particularly on the right side of the colon. ${ }^{10,11}$ While these results likely would not have changed the absolute rate of screening endorsed by physicians in the survey, it may have increased the enthusiasm for strategies not exclusively based on colonoscopy. For example, in the vignettes involving the older and sicker patients, perhaps the tendency to recommend less invasive testing would be even greater if the survey were repeated today.

So, the survey work by Haggstrom suggests that enthusiasm for screening, particularly in the elderly and infirm, may be greater than it should be. What then should be the path forward to minimize the frequency with which patients unlikely to benefit from screening are in fact tested? First, when making decisions about screening, clinicians should consider the absolute benefit of such testing. Undeniably, colorectal cancer is common and lethal. It remains the second most common cause of cancer death in the United States. Encouragingly, significant inroads in reducing the burden of disease have been made, and primary care providers utilizing screening get much of the credit. $^{12}$ That being said, the lifetime risk of colorectal cancer in average risk individuals is only $6 \%$. Therefore, most are never destined to get the disease and can only be harmed by such testing. Moreover, as effective as colorectal cancer screening is, it has never been shown to reduce overall mortality. This was shown most directly in a metaanalysis of nearly 250,000 individuals participating in three large randomized controlled trials of FOBT screening. ${ }^{13}$ While screening reduced colorectal cancer mortality by $13 \%(\mathrm{RR}=0.87,95 \% \mathrm{CI}=0.8-, 0.95)$ there was no benefit in overall mortality $(\mathrm{RR}=1.002,95 \% \mathrm{CI}=0.989-1.015)$. In fact, there was a slight tendency to higher noncolorectal cancer-related mortality in the screened group that could conceivably be related to increased testing ( $R R=1.02,95 \%$ $\mathrm{CI}=1.00-1.04)$. In short, the likelihood that even a healthy 82-year-old will benefit substantially (i.e. live longer) because of a screening stool card or colonoscopy is extremely unlikely.

The second message to clarify about screening is that the decision is a dichotomous one. While a panel of tests is recommended for screening, the intent of that panel is to allow the patient to choose the best option for them. The work by Haggstrom and colleagues suggests that there may be a tendency to offer less invasive tests to those that are older and sicker in the hopes that the result will be negative and not require an invasive test. As an endoscopist, I have often been asked what to do next when a very old or very sick patient tested FOBT positive on a routine screen. At that juncture, knowing the positive result, the decision making becomes increasingly difficult. The time to have the frank discussion about the real risks (e.g. sedation) and benefits of screening in those of advanced age or significant disease needs to occur before any screening is done, not after a noninvasive test suggests there may be a problem.

The third message about screening is that we need to avoid the temptation to offer it simply because an individual is frequently visiting the office or clinic. In the paper by Walter and colleagues exploring VA practice cited above, ${ }^{6}$ one of the strongest predictors of getting screened was having frequent visits to the clinic, and this was independent of comorbidity. Not surprisingly, then, it seems that a major drive to screen is simply encountering the patient. Unfortunately, that approach may have unintended consequences. Oftentimes, those individuals that are coming to the doctor the most frequently are the sickest with the most active medical problems and least likely to benefit from screening. Currently it is estimated that there are 22 million unscreened adults in the United States. ${ }^{14}$ From a public health standpoint, our efforts would be better spent trying to locate those younger and healthier individuals that have never been screened, rather than offering screening to the very old or very sick simply because they are frequently encountered and are readily available to be tested.

Corresponding Author: Douglas J. Robertson, MD, MPH; Section of Gastroenterology (111E), Department of Veterans Affairs Medical Center, White River Junction, VT 05009, USA (e-mail: douglas.robertson@va.gov).

\section{REFERENCES}

1. Haggstrom DA, Klabunde CN, Smith JL, Yuan G. Variation in Primary Care Physicians' colorectal cancer screening recommendations by patient age and comorbidity. J Gen Intern Med. doi:10.1007/s11606012-2093-6.

2. Rex DK, Johnson DA, Anderson JC, Schoenfeld PS, Burke CA, Inadomi JM. American College of Gastroenterology guidelines for colorectal cancer screening 2009 [corrected]. Am J Gastroenterol. 2009;104(3):739-750. 
3. Screening for colorectal cancer: U.S. Preventive Services Task Force recommendation statement. Ann Intern Med. Nov 4 2008;149(9):627-637.

4. Levin B, Lieberman DA, McFarland B, et al. Screening and surveillance for the early detection of colorectal cancer and adenomatous polyps, 2008: a joint guideline from the American Cancer Society, the US Multi-Society Task Force on Colorectal Cancer, and the American College of Radiology. Gastroenterology. 2008;134(5):1570-1595.

5. Sirovich BE, Welch HG. Cervical cancer screening among women without a cervix. J Am Med Assoc. 2004;291(24):2990-2993.

6. Walter LC, Lindquist $\mathbf{K}$, Nugent $\mathbf{S}$, et al. Impact of age and comorbidity on colorectal cancer screening among older veterans. Ann Intern Med. 2009; 150(7):465-473.

7. Schoen RE, Pinsky PF, Weissfeld JL, et al. Colorectal-cancer incidence and mortality with screening flexible sigmoidoscopy. N Engl J Med. 2012;366(25):2345-2357.

8. Segnan N, Armaroli P, Bonelli L, et al. Once-nly sigmoidoscopy in colorectal cancer screening: follow-up findings of the Italian randomized controlled trial-SCORE. J Natl Cancer Inst. 2011;103(17):13101322 .
9. Atkin WS, Edwards R, Kralj-Hans I, et al. Once-only flexible sigmoidoscopy screening in prevention of colorectal cancer: a multicentre randomised controlled trial. Lancet. 2010;375(9726):1624-1633.

10. Baxter NN, Goldwasser MA, Paszat LF, Saskin R, Urbach DR, Rabeneck L. Association of colonoscopy and death from colorectal cancer. Ann Intern Med. 2009;150(1): 1-8.

11. Brenner H, Hoffmeister M, Arndt V, Stegmaier C, Altenhofen L, Haug U. Protection from right-and left-sided colorectal neoplasms after colonoscopy: population-based study. J Natl Cancer Inst. 2010;102(2):89-95.

12. Edwards BK, Ward E, Kohler BA, et al. Annual report to the nation on the status of cancer, 1975-2006, featuring colorectal cancer trends and impact of interventions (risk factors, screening, and treatment) to reduce future rates. Cancer. 2010;116(3):544-573.

13. Moayyedi P, Achkar E. Does fecal occult blood testing really reduce mortality? A reanalysis of systematic review data. Am J Gastroenterol. 2006;101(2):380-384.

14. Vital signs: Colorectal cancer screening, incidence, and mortality-United States, 2002-2010. MMWR Morb Mortal Wkly Rep. Jul 8 2011;60(26):884889 